\title{
A Lipid-Based Depot Formulation with a Novel Non-lamellar Liquid Crystal Forming Lipid
}

\author{
Akie Okada ' • Hiroaki Todo' • Shoko Itakura' • Ichiro Hijikuro ${ }^{2} \cdot$ Kenji Sugibayashi ${ }^{1}$
}

Received: 2 December 2020 / Accepted: 8 February 2021 / Published online: 26 February 2021

(C) The Author(s), under exclusive licence to Springer Science+Business Media, LLC part of Springer Nature 2021

\begin{abstract}
Purpose Non-lamellar liquid crystal (NLLC)-forming lipids have gained attention as a novel component because of their ability to self-assemble upon contact with body fluids. In this study, a novel NLLC-forming lipid, mono-O-(5, 9, 13-trimethyl-4-tetradecenyl) glycerol ester (C17MGE), and a model drug with a middle molecule weight, leuprolide acetate (LA), were used to confirm the usefulness of C17MGE as an excipient for depot formulations with sustained release properties. Methods A self-constructed depot formulation was prepared by mixing G17MGE and different types of phospholipids. The constructed NLLC structure was evaluated using small angle $\mathrm{X}$-ray analysis and cryo-transmission electron microscopy. In vitro release and blood concentration profiles of LA were investigated.
\end{abstract}

Results The NLLC structure was confirmed by small angle $\mathrm{X}$-ray analysis. LA release was able to be modified by adding different ratios of various phospholipids to C17MGE. Formulations containing 1, 2-dioleoyl-sn-glycero-3-phosphoglycerol sodium salt with a mixing ratio of $12 \%$ or $24 \%$ ( $\mathrm{M}_{\mathrm{DOPG12}}$ or $\mathrm{M}_{\mathrm{DOPG} 24}$, respectively) exhibited sustained release profiles of LA. In addition, the blood concentration of LA was detected over 21 days or more after administration of $\mathrm{M}_{\mathrm{DOPG12}}$, and the absolute bioavailability was calculated to be about $100 \%$.

Conclusions A depot formulation using G17MGE was useful to achieve sustained release of LA.

Hiroaki Todo

ht-todo@josai.ac.jp

Faculty of Pharmacy and Pharmaceutical Sciences, Josai University, I - I Keyakidai, Sakado, Saitama 350-0295, Japan

2 Farnex Co., Inc., Tokyo Institute of Technology Yokohama Venture Plaza, 4259-3 Nagatsuta, Midori-ku, Yokohama 226-85 I 0, Japan
KEY WORDS Lipid-based depot formulation · non-lamellar liquid crystal · self-assembled structure · sustained release

\section{INTRODUCTION}

In recent years, the working-age population in developed countries has been declining due to the super-aging of the population (1). Therefore, ensuring working hours for working generations is an important issue. In addition, amidst the novel coronavirus (COVID-19) pandemic situation, many patients hesitate to visit hospital and see their doctor. The development of excipients that enable sustained-release delivery of drugs may improve the quality of life of patients and reduces the risk of recurrence or deterioration of symptoms. In addition, such delivery systems may be helpful to reduce the burden on healthcare professionals. Nowadays, middle-sized molecules (M.W. ca 1-5 kDa) including peptides and nucleic acids are expected as the next target therapeutic drugs because of their high affinity and selectivity to disease sites. Thus, it would be useful to develop new excipients or vesicles that enable sustained delivery for various kinds of therapeutic drugs.

Many reports have been published in the last 5 years showing the usefulness of non-lamellar liquid crystals (NLLCs) as pharmaceutical excipients to obtain sustained drug release (2). NLLCs increases the solubility of poorly soluble drugs and improve the stability of the higher-order structure of proteins (3-5). Ki et al. (6) reported an injectable NLLC system composed of sorbitan monooleate (known as Span 80), phosphatidylcholine, and tocopherol acetate. The NLLC formulation showed sustained release of leuprolide acetate (LA) over 1 month and exhibited a similar bioavailability to the commercial subcutaneous (s.c.) depot formulation. In particular, lipids that form an NLLC structure upon contact with body fluids would be useful as injectable excipients. A lipid-based structure is an excellent alternative to polylactic acid-glycolic 
acid copolymer (PLGA) depot formulations. The stability of peptides and proteins entrapped in the depot formulation is a critical point for long-term drug delivery (7). In the PLGA formulation, the acidic products resulting from the PLGA degradation may affect the drug stability by lowering the $\mathrm{pH}$ in the formulation (8). Therefore, injectable lipid-based depot formulations may be a promising platform and enable desired drug release and adequate shelf-life longevity.

As mentioned above, NLLC-forming lipids may be attractive pharmaceutical excipient for depot formulations. However, only a limited number of NLLC-forming lipids have been developed, such as glycerol monooleate (GMO), glycerol dioleate, and phytantoriol (PHY) $(9,10)$. In the present study, we focused on a newly developed NLLC-forming lipid, mono-O-(5, 9, 13-trimethyl-4-tetradecenyl) glycerol ester (C17MGE), which exists as a liquid state at room temperature and has a low viscosity $(0.47 \mathrm{~Pa} \cdot \mathrm{s}$ at a share rate of $10 \mathrm{~s}^{-1}$ ) compared with GMO in a room temperature $(11,12)$.

In our previous report, a newly prepared depot formulation with C17MGE successfully showed an NLLC structure in the body after s.c. injection into rats, and the release rate of a hydrophilic middle molecular weight compound, fluorescein isothiocyanate-dextran (M.W.; 4000, FD-4), was controlled by alternation of the interplanar spaces and NLLC structure by adding several types of phospholipids (13). This smart phase transition with C17MGE suggested that it may be a promising drug depot base with sustained release properties of entrapped drugs.

In the present study, LA, a linear nonapeptide with a positive charge at neutral $\mathrm{pH}\left(p K_{a}\right.$ approximately 6.0: the imidazolyl nitrogen of histidine) (14), was selected as a model middle molecular weight drug. LA is unstable in the skin tissue (15). Therefore, a sustained-release formulation with a high bioavailability of LA is necessary to show the usefulness of C17MGE-based depot formulations. A self-assembled structure formed by C17MGE was evaluated by adding several types of phospholipids, and LA release from the constructed structure and blood-concentration profile were investigated to confirm the usefulness of C17MGE as the excipient for a longacting depot formulation.

\section{MATERIALS AND METHODS}

\section{Materials}

LA was purchased from Shin Nippon Yakugyo Co Ltd. (Tokyo, Japan). C17MGE was provided by Farnex Inc. (Yokohama, Japan). Table I shows the chemical structures of LA and C17MGE. Among phospholipids, 1, 2-distearoyl-snglycero-3-phosphocholine (DSPC) was selected as a saturated example and 1, 2-dioleoyl-sn-glycero-3-phosphoglycerol, sodium salt (DOPG), 1, 2-dioleoyl-sn-glycero-3- phosphoethanolamine (DOPE), and phosphocholine (DOPG) were selected as unsaturated phospholipids. These phospholipids were purchased from NOF Corporation (Tokyo, Japan). Table II shows the chemical structure of these phospholipids. This table also shows the abbreviations used for the phospholipids. Polyoxyethylene sorbitan monooleate (Tween 80) was purchased from Tokyo Chemical Industries, Ltd. (Tokyo, Japan). All other regents were used without further purification.

\section{Preparation of Formulations}

Table III shows the composition of the prepared depot formulations. C17 MGE, ethanol, and Tween 80 were weighed and added to a $9 \mathrm{~mL}$ vial (laboratory screw tube bottle No. 3, AS ONE K.K., Osaka, Japan) containing a PTFE stirrer (Double Cross-Type D-10 F-4037-01, CFC Corporation, Tokyo, Japan). The weighed compounds were heated at $60^{\circ} \mathrm{C}$ on a hot plate (Hotplate Stirrer RSH-1DN, AS ONE K.K.) while stirring at $500 \mathrm{rpm}$ for $5 \mathrm{~min}$. Then, weighed saturated or unsaturated phospholipid was added and further mixed at $60^{\circ} \mathrm{C}$ while stirred at $700 \mathrm{rpm}$ for $60 \mathrm{~min}$. The prepared formulations shown in Table II were abbreviated to $\mathrm{M}_{\alpha \beta}$; where $\alpha$ is the type of phospholipid (DSPC/DOPC/ DOPE/DOPG) and $\beta$ is the phospholipid content (i.e., $\mathrm{M}_{\mathrm{DSPC} 12}$ is a formulation containing $76 \% \mathrm{C} 17 \mathrm{MGE}$ and $12 \%$ DSPC in the formulation). In addition, C17 MGE alone (abbreviated as $\mathrm{M}_{\text {only }}$ ) and C17 MGE with ethanol and Tween 80 (abbreviated as M) were prepared for comparison. Finally, LA was mixed with the prepared formulations using a Pellet Pestle (Thermo Fisher Scientific K.K., Tokyo, Japan) for $2 \mathrm{~min}$. LA concentration in the formulation was $37.5 \mathrm{mg} /$ $\mathrm{mL}$.

\section{Small Angle X-Ray Analysis}

The prepared formulations were analyzed using a small angle X-ray diffraction instrument (Nano-Viewer, Rigaku Co., Ltd., Akishima, Tokyo, Japan) (CuK $\alpha$ radiation, $\lambda=1.5418 \AA$ ) operated at $30 \mathrm{kV}$ and $40 \mathrm{~mA}$. The camera focal length was set to $700 \mathrm{~mm}$. Formulations immersed into $3 \mathrm{~mL}$ of phosphate-buffered saline (PBS) for $6 \mathrm{~h}$ were used as samples, and measurements were performed by Kanazawa University (Kanazawa, Ishikawa, Japan). The measurement conditions were as described previously (16). Crystalline interplanar spacing, $d$, was determined in accordance with the Bragg equation.

\section{In Vitro Release Test from the Formulation}

The prepared formulation containing LA $(100 \mu \mathrm{L})$ was added to dialysis tubing (Pur-A-Lyzer ${ }^{\text {TM }}$ Mini 12,000 dialysis kit 25, molecular cut-off 12,000, Sigma Aldrich, St. Louis, MO, U.S.A.), then the dialysis tube was placed into a $25 \mathrm{~mL}$ 
Table I. Structures of LA and MGE

Drug
glycerol ester
(C17MGE)

centrifuge tube (centrifuge tube Mini MINI-2362-025, AGC Technoglass Co., Ltd., Shizuoka, Japan) containing $20 \mathrm{~mL}$ of PBS with $0.02 \%$ Tween 80 . LA release experiments were conducted using the dialysis method.
During the release experiment, the centrifuge tube, covered with aluminum foil to prevent LA degradation, was set in a water bath (AS ONE Corporation, Osaka, Japan) at $37 \pm$ $0.02^{\circ} \mathrm{C}$. The outer solution was periodically sampled over 7 days. The same amount of fresh PBS containing $0.02 \%$

Table II. Structure of Phospholipids

\begin{tabular}{|c|c|c|}
\hline Drug & Structures & ${ }^{*} X \log P 3$ \\
\hline $\begin{array}{l}\text { 1, 2-Distearoyl-sn-glycero-3- } \\
\text { phosphocholine } \\
\text { (DSPC) }\end{array}$ & 0 & 13.2 \\
\hline $\begin{array}{l}\text { 1, 2-Dioleoyl-sn-glycero-3- } \\
\text { phosphocholine } \\
\text { (DOPC) }\end{array}$ & & 12.8 \\
\hline $\begin{array}{l}\text { 1, } \\
\text { 2-Dioleoyl-sn-glycero-3-pho } \\
\text { (DOPE) }\end{array}$ & & 10.6 \\
\hline $\begin{array}{l}\text { 1, 2-Dioleoyl-sn-glycero-3- } \\
\text { phosphoglycerol, } \\
\text { sodium salt } \\
\text { (DOPG) }\end{array}$ & & \\
\hline
\end{tabular}


Table III Composition of Prepared Depot Formulations

Maß

\begin{tabular}{|c|c|c|c|c|c|c|c|c|c|c|}
\hline \multirow{3}{*}{$\begin{array}{l}\alpha \\
\beta\end{array}$} & \multirow{2}{*}{\multicolumn{2}{|c|}{$\begin{array}{ll} & \\
& -\end{array}$}} & \multicolumn{2}{|c|}{$M_{D S P C}$ formulations } & \multicolumn{2}{|c|}{ MDOPC formulations } & \multicolumn{2}{|c|}{ M DOPE formulations } & \multicolumn{2}{|c|}{$M_{D O P G}$ formulations } \\
\hline & & & \multicolumn{2}{|c|}{ DSPC } & \multicolumn{2}{|c|}{ DOPC } & \multicolumn{2}{|c|}{ DOPE } & \multicolumn{2}{|c|}{ DOPG } \\
\hline & - & - & 12 & 24 & 12 & 24 & 12 & 24 & 12 & 24 \\
\hline Formulation name & Monly & $M$ & $M_{D S P C 12}$ & $M_{D S P C 24}$ & $M_{D O P C 12}$ & $M_{\text {DOPC24 }}$ & MDOPEI2 & MDOPE24 & $M_{D O P G 12}$ & $M_{D O P G 24}$ \\
\hline CI7MGE & 100 & 88 & 76 & 64 & 76 & 64 & 76 & 64 & 76 & 64 \\
\hline DSPC & & - & 12 & 24 & - & - & - & - & - & - \\
\hline DOPC & & - & - & - & 12 & 24 & - & - & - & - \\
\hline DOPE & & - & - & - & - & - & 12 & 24 & - & - \\
\hline DOPG & & - & - & - & - & - & - & - & 12 & 24 \\
\hline Ethanol & & & & & & 10 & & & & \\
\hline Tween 80 & & & & & & 2 & & & & \\
\hline
\end{tabular}

unit: percentage

Tween 80 was added to the outer compartment to maintain a constant volume. The outer solution was agitated using a pipet for $10 \mathrm{~s}$ prior to each sampling. The obtained samples were stored at $-80^{\circ} \mathrm{C}$ until measurement. The cumulative percentage of LA released was calculated using the amount of LA loaded in the formulation.

\section{In Vivo Experiments}

Male Wistar rats (body weight $200 \pm 20$ g, 8 weeks old) were purchased from Sankyo Lab Services, Inc. (Tokyo, Japan). Rats were housed in a room maintained at $25 \pm 2{ }^{\circ} \mathrm{C}$ with a 12-h-dark/light cycle (on, off time: 9:00, 21:00). In addition, drinking water and feed (MF, Oriental Yeast Co., Ltd., Tokyo, Japan) were supplied ad libitum. The experiments were conducted in accordance with the experimental animal regulations of Josai University after obtaining consent (JU19009) from the Ethical Commission of Josai University.

Rats were cannulated into the jugular vein. After resting for 1 day post-surgery, the prepared formulation was administered subcutaneously via a $23 \mathrm{G}$ needle into the dorsal region. The administration site was shaved before administration. Blood sampling $(100 \mu \mathrm{L})$ was conducted periodically until 28 days after administration from the cannulation tube. The same amount of saline was injected into the rats through the cannulation tube after every sampling. The blood samples were centrifuged $\left(21,500 \times \mathrm{g}, 5 \mathrm{~min}, 4^{\circ} \mathrm{C}\right)$ to obtain plasma. The obtained plasma was stored at $-80^{\circ} \mathrm{C}$ until measurement. The lower limit in the quantitative detection of LA was $1 \mathrm{ng} / \mathrm{mL}$ in the present conditions. The area under the curve over 21 days after administration $\left(A U C_{21 \text { days }}\right)$ was calculated using the trapezoidal method. The extent of bioavailability $(F)$ was calculated as the ratio of $A U C_{21 \text { days }}$ after s.c. and intravenous (i.v.) administrations. LA solution dissolved in saline at a concentration of $4 \mathrm{mg} / \mathrm{mL}$ was administered through the jugular vein at a dose of $5 \mathrm{mg} / \mathrm{kg}$, whereas the prepared formulation was administered s.c. at a dose of $18.75 \mathrm{mg} / \mathrm{kg}$.

\section{LA Determination}

Samples obtained in the in vitro release experiment were mixed with acetonitrile at a 1:1 $(\mathrm{v} / \mathrm{v})$ ratio and vortexed for $5 \mathrm{~min}$ and then used as the measured sample. Plasma obtained in the in vivo experiment was vortexed with acetonitrile at a ratio of $1: 1(\mathrm{v} / \mathrm{v})$ for $5 \mathrm{~min}$ and then centrifuged $\left(21,500 \times \mathrm{g}, 5 \mathrm{~min}, 4^{\circ} \mathrm{C}\right)$ to use the upper layer as a measured sample.

The liquid chromatography-tandem mass spectrometry (LC-MS/MS) system consisted of a system controller (CBM20A; Shimadzu Corporation, Kyoto, Japan), pump (LC20 AD; Shimadzu Corporation), auto-sampler (SIL-20 AC; Shimadzu Corporation), column oven (CTO-20 AC; Shimadzu Corporation), detector (3200 QTRAP; AB Sciex, Tokyo, Japan), and analysis software (Analyst ${ }^{\circledR}$ version 1.4.2; Shimadzu Corporation). The column and the guard column were Shodex ${ }^{\circledR}$ ODP2 HP-2B $2.0 \mathrm{~mm} \times 50 \mathrm{~mm}$ and ODP2 HPG-2A $2.0 \mathrm{~mm} \times 10 \mathrm{~mm}$, respectively (Showa Denko, Tokyo, Japan). The column temperature was adjusted to $40^{\circ} \mathrm{C}$. An internal standard of betamethasone valerate was used for the LA assay. A mixed solution (A:B at a 70:30 ratio) was used for the mobile phase, where $A$ was $0.1 \%$ formic acid in water, and $\mathrm{B}$ was acetonitrile. The flow rate was $0.2 \mathrm{~mL} /$ min, and the injection volume was set to $10 \mu \mathrm{L}$. Electrospray ionization was used for LA ionization. The measured molecular weight of LA was set to m/z 605.30 for the precursor ion and $\mathrm{m} / \mathrm{z} 249.00$ for the product ion. The ion spray voltage was $5000 \mathrm{~V}$, the nebulizer gas pressure was 80 psi, the drying gas flow rate was $10 \mathrm{~L} / \mathrm{min}$, and the drying gas temperature was $600^{\circ} \mathrm{C}$. The lower limit of quantification of this assay was $1.0 \mathrm{ng} / \mathrm{mL}$. 


\section{Observation with a Cryo-Transmission Electron Microscope}

The formulation immersed into $3 \mathrm{~mL}$ of PBS was observed using a cryo-transmission electron microscope (Cryo-TEM) (JEM-3100FEF, JEOL Ltd., Akishima, Tokyo, Japan). For imaging, $1 \mu \mathrm{L}$ of a 20 -fold dilution of the formulation was dropped onto a hydrophilized copper grid (200 mesh, JEOL Corporation) and blotted. The samples were rapidly frozen using ethane as a freezing solvent using a rapid freezing system (EM-CPC, Leica Microsystems Japan, Tokyo, Japan) for observation using the Cryo-TEM at 5-10 $\mu \mathrm{m}$ defocus.

\section{Statistical Analysis}

All experiments, except for SAXS analysis, were conducted with more than three replicates per formulation. JMPß Pro software (ver. 14.0.0, SAS Institute Inc., Cary, NC, U.S.A.) was used for statistical analyses. Experimental data were tested for statistical significance $(p<0.05)$ using one-way ANOVA and Tukey's honestly significant difference post hoc analysis. All data were expressed as mean with standard deviation.

\section{RESULTS}

\section{Small Angle X-Ray Analysis of the Prepared Formulation}

Figure 1 shows the small angle X-ray diffraction peak for each formulation containing LA, and Table IV shows the crystal structure obtained by analyzing the diffraction peak and the inter-pore distances $(d)$ calculated from Bragg's equation. Figure $1 \mathrm{a}-\mathrm{d}$ shows the results for $\mathrm{M}_{\mathrm{DSPC}}, \mathrm{M}_{\mathrm{DOPC}}, \mathrm{M}_{\mathrm{DOPE}}$, and $\mathrm{M}_{\mathrm{DOPG}}$ formulations, respectively. Formulations $\mathrm{M}_{\text {only, }}$, $\mathrm{M}_{\text {DOPE 12, }}$, and $\mathrm{M}_{\text {DOPE24 }}$ exhibited reverse-hexagonal $\left(H_{2}\right)(1$ : $\sqrt{ } 3: \sqrt{4})$ and cubic structures with $\operatorname{Pn} 3 m$ space groups $(\sqrt{2}: \sqrt{ } 3$ : $\sqrt{4}$ ), whereas $\mathrm{M}, \mathrm{M}_{\mathrm{DSPC} 12}, \mathrm{M}_{\mathrm{DSPC} 24}, \mathrm{M}_{\mathrm{DOPC} 12}, \mathrm{M}_{\mathrm{DOPC} 24}$, $\mathrm{M}_{\text {DOPG12, }}$, and $\mathrm{M}_{\mathrm{DOPG} 24}$ displayed only cubic structures with Pn3m space groups $(\sqrt{ } 2: \sqrt{ } 3: \sqrt{ } 4: \sqrt{6}: \sqrt{8}: \sqrt{ } 9)$. Shifted diffraction peaks toward lower diffraction angles were observed in the $\mathrm{M}_{\mathrm{DSPC}}, \mathrm{M}_{\mathrm{DOPC}}, \mathrm{M}_{\mathrm{DOPG}}$, and $\mathrm{M}_{\mathrm{DOPE}}$ formulations compared with M. Effect of the addition of LA in the formulation was also investigated (Table IV, SAXS data not shown). The presence of LA induced structural transformation, except for $\mathrm{M}_{\mathrm{DOPG}}$ formulations.

Figure 2 shows representative cryo-TEM images of NLLC constructed formulations. Cryo-TEM images confirmed the characteristic multilayer pattern of the $\mathrm{H}_{2}$ structure in $\mathrm{M}_{\text {only }}$ (a) and the presence of the ordered pattern of cubic phase in $\mathrm{M}$ (b) and $\mathrm{M}_{\mathrm{DOPG} 24}$ (c).

\section{In Vitro Release Experiments from the Prepared Formulations}

Figure 3 shows the release profile of LA from the prepared depot formulations against the square root of time. Linear lines were confirmed with each LA release. When DSPC and DOPE were included in the formulation $\left(\mathrm{M}_{\mathrm{DSPC}}\right.$ and $\mathrm{M}_{\text {DOPE }}$, respectively), LA release was higher than from $\mathrm{M}$. Higher release ratios ( $>40 \%$ after 7 days) were obtained from Msc and Moe formulations despite the phospholipid content. On the other hand, DOPG-containing formulations $\left(\mathrm{M}_{\mathrm{DOPG} 12}\right.$ and $\left.\mathrm{M}_{\mathrm{DOPG} 24}\right)$ showed lower LA release over 7 days, and only about $20 \%$ of the cumulative amount of released LA was observed from the $\mathrm{M}_{\mathrm{DOPG}}$ formulations.

\section{LA Profile in Blood after s.c. injection of the Prepared Formulations}

Figure 4 shows the changes in LA plasma concentration after s.c. administration of the depot preparations to a dorsal site in rats. In this experiment, the $\mathrm{M}_{\mathrm{DOPG}}$ formulations were only administered in phospholipid-containing formulations because they showed lower LA release compared with the other formulations. LA solution and $\mathrm{M}$ were also administered to investigate the effect of C17MGE and DOPG in the formulation on the blood concentration profile of LA.

LA was rapidly eliminated from the blood, and no detection was confirmed about $6 \mathrm{~h}$ after the s.c. administration of LA solution. On the other hand, LA was detected in blood over 21 days after administration of $\mathrm{M}$ and $\mathrm{Mog}$ formulations. In particular, the $\mathrm{M}_{\mathrm{DOPG} 12}$ formulation provided a significantly higher LA concentration in blood compared with M at 14 days after administration.

Table $\mathrm{V}$ summarizes the pharmacokinetic parameters $\left(A U C_{21 \text { days }}, F\right)$ for each formulation. AUC was calculated using the trapezoidal rule over 21 days after administration. The $F$ value obtained with the s.c. administration of LA solution was $4.31 \%$, whereas those of the formulations containing MGE were significantly higher $(p<0.01)$ than that obtained using the LA solution. In particular, the $\mathrm{M}_{\mathrm{DOPG} 12}$ formulation exhibited the highest $F$ value $(89.1 \%)$ among the MGE contained formulations.

\section{DISCUSSION}

Lipid-based depot formulations have been actively studied in the last 5 years. In particular, NLLC-forming lipids have gained attention as novel excipients for depot formulations because they conveniently construct a depot upon contact with body fluids. The usefulness of a novel lipid, G17MGE, has been investigated, and it was found that formulations composed of C17MGE can take advantage of transdermal 
Fig. I Structural analysis of constructed gel by small angle X-ray diffraction. The constructed gel was obtained by dropping the depot formulations of $M_{D S P C}(\mathbf{a}), M_{D O P C}$ (b), $M_{D O P E}(\mathbf{c})$ and $M_{D O P G}(\mathbf{d})$ into PBS. Symbols: •; peak derived from reverse hexagonal structure, $\Delta$; peak derived from cubic structure with Pn3m space group. (a)
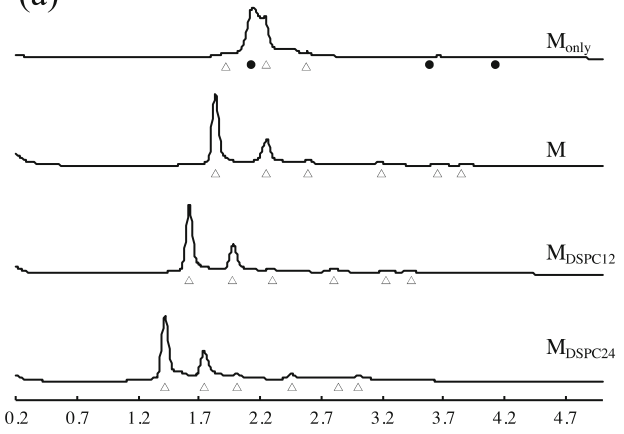

(c)

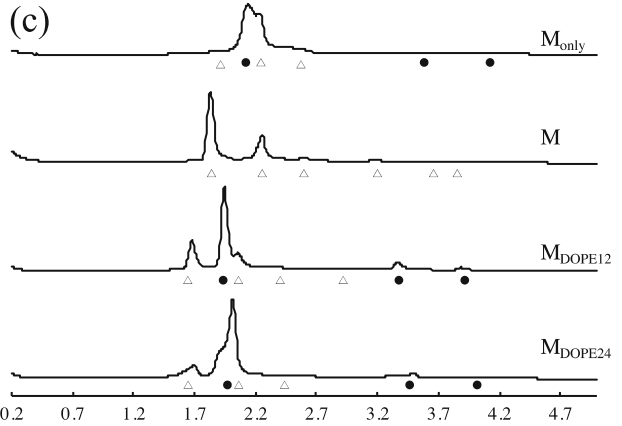

(b)

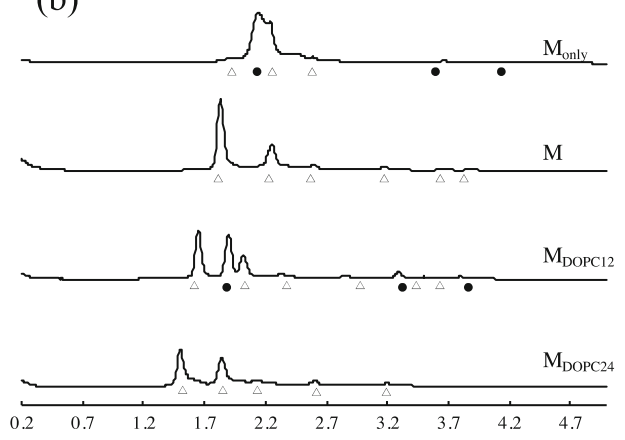

(d)

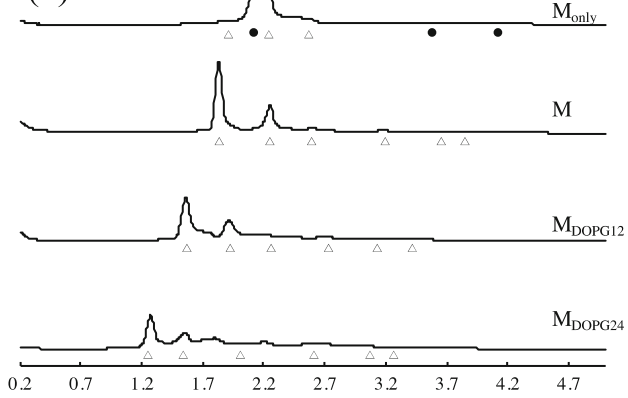

$(11,12)$, nose-to-brain (13), oral mucosal (17), and oral (18) delivery routes compared with a conventional, commercial products. In addition, a controlled drug release system based on the NLLC structure was found to be available with

Table IV Constructed Structure and its Interplanar Spacing $(d, \mathrm{~nm})$ of Prepared Formulations with or Without LA

\begin{tabular}{|c|c|c|}
\hline Formulation & Liquid crystal structure & $d(\mathrm{~nm})$ \\
\hline Monly & $\begin{array}{l}\mathrm{H}_{2}+\mathrm{Pn} 3 \mathrm{~m} \\
\left(\mathrm{H}_{2}\right)\end{array}$ & $\begin{array}{l}4.10 \\
(4.36)\end{array}$ \\
\hline M & $\begin{array}{l}\mathrm{Pn} 3 \mathrm{~m} \\
\left(\mathrm{H}_{2}\right)\end{array}$ & $\begin{array}{l}4.82 \\
(4.36)\end{array}$ \\
\hline$M_{\mathrm{DSPCI} 2}$ & $\begin{array}{l}P n 3 m \\
\left(H_{2}+P n 3 m\right)\end{array}$ & $\begin{array}{l}5.46 \\
(4.87)\end{array}$ \\
\hline$M_{\mathrm{DSPC24}}$ & $\begin{array}{l}\mathrm{Pn} 3 \mathrm{~m} \\
\left(\mathrm{H}_{2}\right)\end{array}$ & $\begin{array}{l}6.22 \\
(5.49)\end{array}$ \\
\hline$M_{\mathrm{DOPC} 12}$ & $\begin{array}{l}\mathrm{Pn} 3 \mathrm{~m} \\
\left(\mathrm{H}_{2}\right)\end{array}$ & $\begin{array}{l}5.35 \\
(4.65)\end{array}$ \\
\hline$M_{\mathrm{DOPC} 24}$ & $\begin{array}{l}\mathrm{Pn} 3 \mathrm{~m} \\
\left(\mathrm{H}_{2}\right)\end{array}$ & $\begin{array}{l}5.87 \\
(4.95)\end{array}$ \\
\hline$M_{\text {DOPE } 12}$ & $\begin{array}{l}\mathrm{H}_{2}+\mathrm{Pn} 3 \mathrm{~m} \\
\left(\mathrm{H}_{2}\right)\end{array}$ & $\begin{array}{l}4.30 \\
(4.55)\end{array}$ \\
\hline$M_{\text {DOPE24 }}$ & $\begin{array}{l}\mathrm{H}_{2}+\mathrm{Pn} 3 \mathrm{~m} \\
\left(\mathrm{H}_{2}\right)\end{array}$ & $\begin{array}{l}4.37 \\
(4.55)\end{array}$ \\
\hline MDOPGI2 & $\begin{array}{l}\text { Pn3m } \\
(\text { Pn3m) }\end{array}$ & $\begin{array}{l}5.65 \\
(5.82)\end{array}$ \\
\hline$M_{\text {DOPG24 }}$ & $\begin{array}{l}\text { Pn3m } \\
(P n 3 m)\end{array}$ & $\begin{array}{l}6.91 \\
(7.31)\end{array}$ \\
\hline
\end{tabular}

The parenthesis shows liquid crystal structure and its $d$ value of the formulation without LA that calculated from SAXS observation results
C17MGE-based formulations (16). Therefore, we investigated the development of C17MGE-based depot formulation that accomplished a sustained release and improved bioavailability of LA, a model middle molecular weight drug, in the present study.

Many reports have been published on controlled drug release with NLLCs. Fong et al. reported temperaturedependent drug release by changing the NLLC structure from a cubic structure to a reverse-hexagonal structure, and vice versa (19). Báez-Santos et al. (20) showed that drug release was increased by alternation of the inter-pore distances in the structure caused by increased water content in NLLC formulations composed of phosphatidylcholine, Span 80, and tocopherol acetate. Tocopherol acetate was added to the formulations as a phase transition modifier. Various other phase transition modifiers have been reported, such as tetradecane, limonene, and triolein (10). However, few reports have studied the influence of phospholipids on the phase behavior and entrapped drug release from NLLC structures. In the present study, different phospholipids were used as phase transition modifiers for C17MGE-based NLLG structures.

Small angle X-ray analysis of the prepared formulations showed that their structure was affected by the type and content of phospholipid in the formulation (Fig. 2). In general, the relationship between the structure of amphiphiles, including phospholipids, and the shape of the self-assembled structures can be considered with the critical packing parameter (CPP) (10). DSPC and DOPC, which were used in this study, are cylindrical in shape with $\mathrm{CPP} \sim 1$ and are known to form 
Fig. 2 Representative cryo-TEM of $M_{\text {only }}(\mathbf{a}), M(\mathbf{b})$, and $M_{\text {DOPG24 }}(\mathbf{c})$ after immersion in PBS for $24 \mathrm{~h}$. Arrows show the presence of a multilayer pattern in (a) and the presence of an ordered pattern (b) and (c). Black bar: $50 \mathrm{~nm}$.
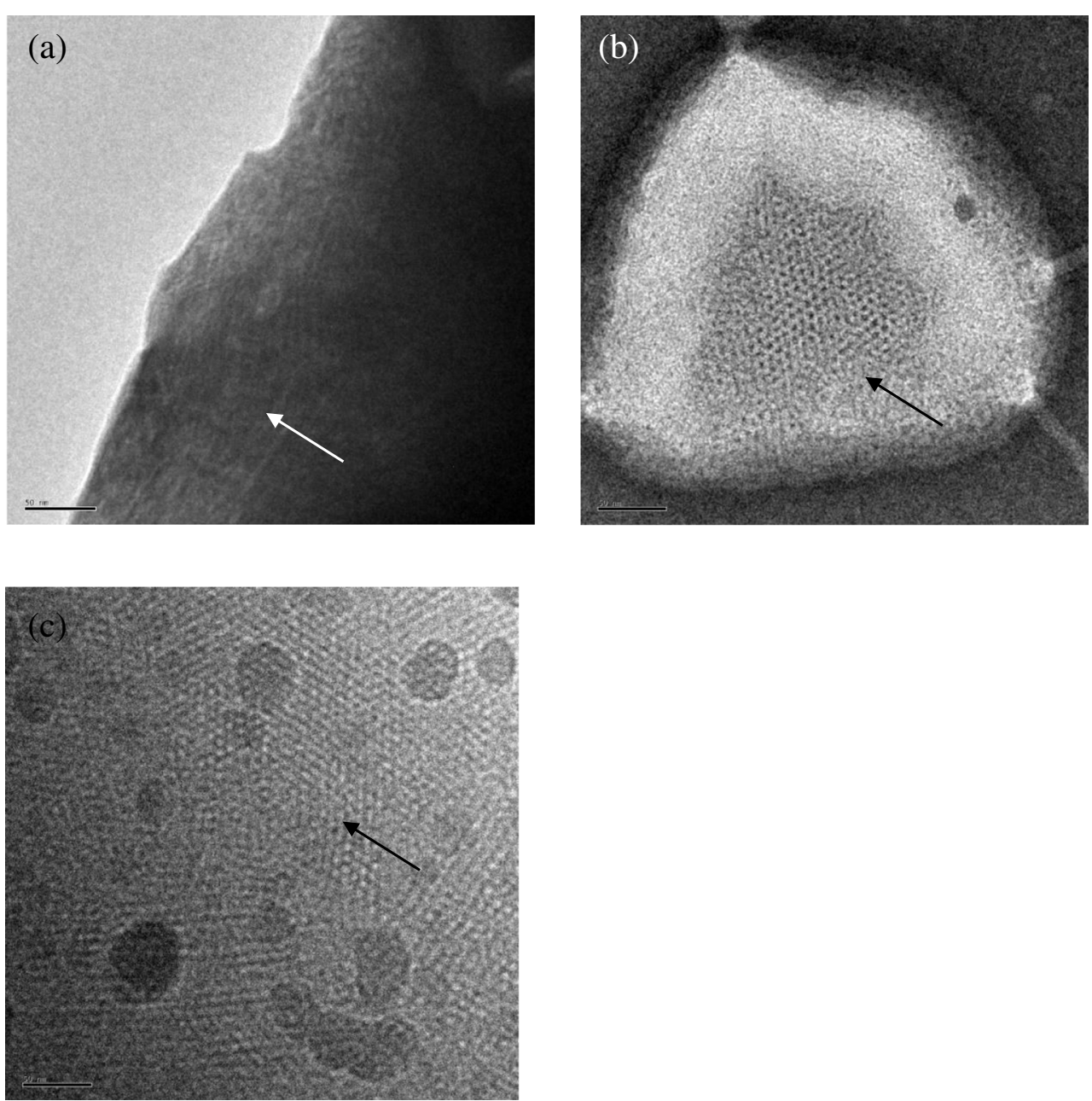

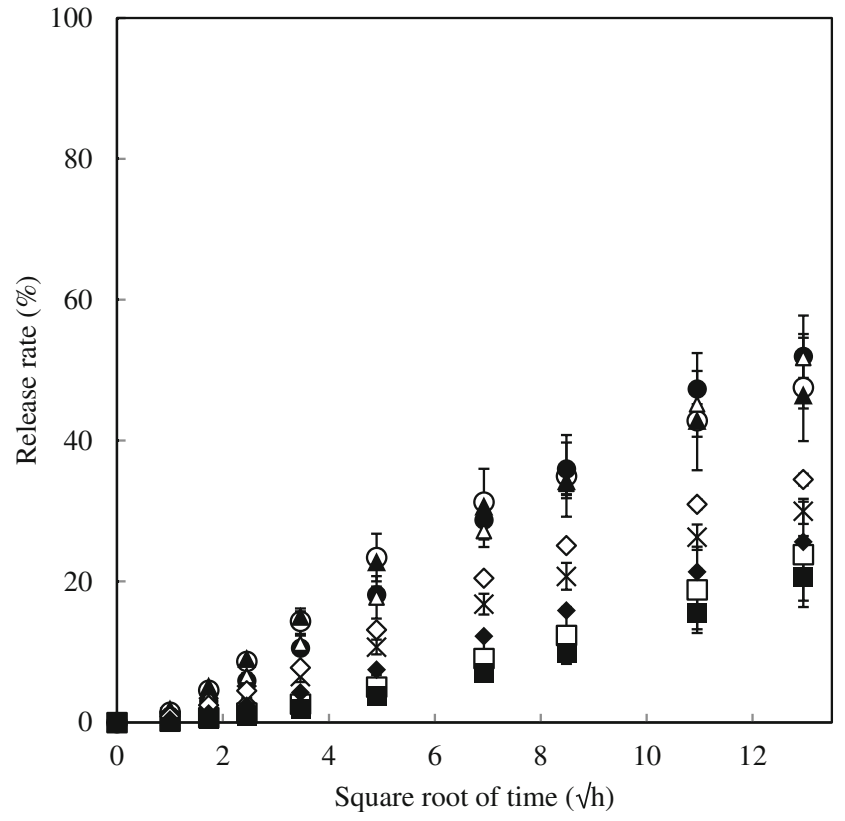

Fig. 3 LA release profile from formulations over 7 days. Symbols: $M(\times)$, $M_{\text {DSPC12 }}(0), M_{\text {DSPC24 }}(\bullet), M_{\text {DOPC12 }}(\downarrow), M_{\text {DOPC24 }}(\bullet), M_{\text {DOPE12 }}(\Delta)$, $M_{\text {DOPE24 }}(\boldsymbol{\Delta}), M_{\text {DOPG I2 }}(\square), M_{D O P G 24}(\boldsymbol{\bullet})$. Each value shows the mean \pm S.D. $(n=3-5)$. bilayer vesicles. DOPE and DOPG have CPP $>1$ due to their relatively small headgroups compared with the other phospholipids. The addition of phospholipids with $\mathrm{CPP} \sim 1$ and $\mathrm{CPP}>1$ in the formulation was thought to provide a phase transition toward lamellar phases and $H_{2}$ phases, respectively, by decreasing and increasing membrane curvature, respectively. However, peak shifts toward the lower angle and increased interplanar spacing were observed by increasing the phospholipid amount without a change in the NLLC structure, except for $\mathrm{M}_{\text {DOPE }}$ formulations (Fig. 2). Yaghmur et al. (21) reported that diglycerol monooleate $(\mathrm{CPP} \sim 1)$ interacted with the polar head group of monolinolein to increase water channel dimension in a monolinolein-based phase structure. Therefore, the addition of phospholipids with $\mathrm{CPP} \sim 1$ may contribute to the induction of increased water channel dimensions. Another factor that decreases membrane curvature is CPP $>1$ with a charged headgroup such as DOPG. A high phospholipid content is thought to contribute to the stabilization and phase transition of the formed NLLC structure. In the present study, phase transition was only observed in the case of increasing negative curvature by the addition of DOPE. 
Fig. 4 Plasma LA concentrationtime profile over 21 days after subcutaneous injection of LA sol. (•), M (०), M DOPG $12_{2}(\square), M_{D O P G 24}(\mathbf{\bullet})$. Each point shows the mean \pm S.E. $(n=3-5)$.

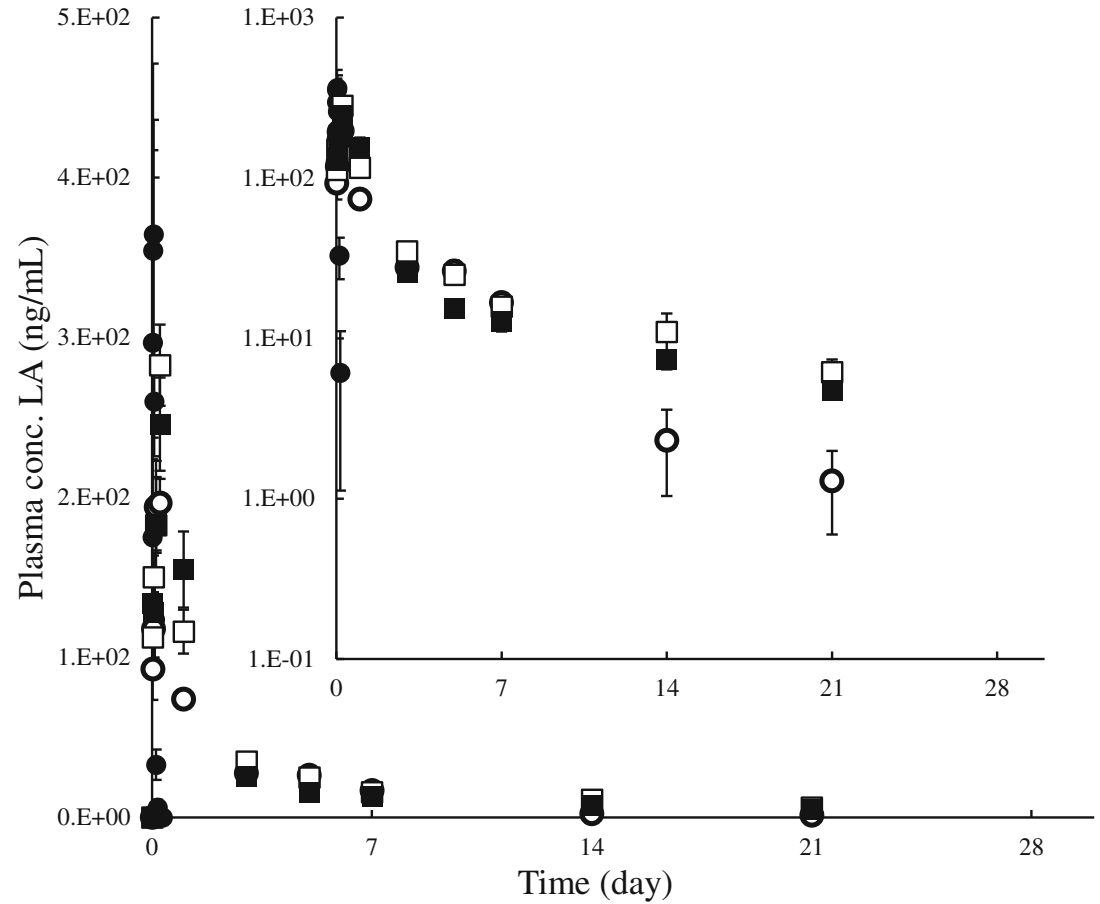

In the present study, no SAXS observation, but a polarizing microscopic observation was performed with collected formulation after 6-12 h from the administration site. Specific polarization images derived from NLLC structure were confirmed (data not shown). Therefore, construction of NLLC structure was thought to be obtained at administration site by body fluid after s.c. injection.

Release rates of entrapped drugs are faster in the order; lamellar phase, cubic phases, hexagonal phases, and micellar cubic $(22,23)$. However, $\mathrm{M}_{\mathrm{DOPE}}$ formulations with an $\mathrm{H}_{2}$ structure showed higher LA release compared with $\mathrm{M}$ and $\mathrm{M}_{\mathrm{DOPC}}$ formulations with $\mathrm{Pn} 3 \mathrm{~m}$ space groups. In our previous study, the release ratio of fluorescein isothiocyanate dextran (M.W., 4000, FD-4), a hydrophilic drug, from a constructed NLLC structure was increased with an increase in interplanar spacing, despite the difference in the constructed

Table V Pharmacokinetic Parameters of Leuprolide Acetate After S.C. CoAdministration in Rats

\begin{tabular}{|c|c|c|c|c|c|c|c|c|c|}
\hline \multirow[t]{2}{*}{ Route } & \multirow{2}{*}{$\begin{array}{l}\text { Dose } \\
(\mathrm{mg} / \mathrm{kg})\end{array}$} & \multirow[t]{2}{*}{ Formulations } & \multicolumn{4}{|c|}{$A \cup C_{21 \text { days }}$} & \multicolumn{3}{|c|}{$F$} \\
\hline & & & \multicolumn{4}{|c|}{$(\mu \mathrm{g} / \mathrm{min} / \mathrm{mL})$} & \multicolumn{3}{|c|}{ (\%) } \\
\hline i.v. & 5 & - & 228.86 & \pm & 27.53 & & - & \pm & - \\
\hline \multirow[t]{4}{*}{ S.C. } & 18.75 & Solution & 36.48 & \pm & 4.57 & & 4.31 & \pm & 0.54 \\
\hline & & $M$ & 540.55 & \pm & 41.75 & * & 63.84 & \pm & 4.93 \\
\hline & & $M_{\text {DOPGI2 }}$ & 754.06 & \pm & 44.47 & * & 89.05 & \pm & 5.25 \\
\hline & & $M_{D O P G 24}$ & 660.71 & \pm & 69.31 & * & 78.03 & \pm & 8.19 \\
\hline \multicolumn{7}{|c|}{$F=\left[\left(\operatorname{AUC}_{\text {s.c. }} / \text { Dose }_{\text {s.c. }}\right) /\left(\mathrm{AUC}_{\text {i.v. }} / \text { Dose }_{\text {i.v. }}\right)\right]^{*} \mid 00$} & & \pm & S.E. \\
\hline
\end{tabular}

* $p<0.0$ I Compared with s.c. administration of LA solution structure (18). On the other hand, no relationship was found between LA released from the formulations and the value of interplanar spacing in the present study (data not shown). Hydrophilic drugs are located in the water channels or close to the polar head, while hydrophobic drugs and amphiphilic drugs are loaded in the lipid layer and at the lipid layer interface, respectively (22). When effect of the addition of LA on the constructed structure was evaluated with SAXS, the structure was affected by the addition of $\mathrm{LA}$, except for $\mathrm{M}_{\mathrm{DOPG}}$ formulation (Table IV). This result suggested that LA interacted with the MEG or/and phospholipid, which in turn caused the phase transition. The interaction would be altered by a hydrophilic group in the MGE or / and phospholipid and filled void volume in the structure. Therefore, these interactions might be a reason for higher $\mathrm{LA}$ release from $\mathrm{M}_{\mathrm{DOPE}}$ formulation compared with $\mathrm{M}$ and $\mathrm{M}_{\mathrm{DOPC}}$ formulations in the present study.

$\mathrm{M}_{\mathrm{DOPG}}$ formulations showed sustained release compared with the other formulations. Figure 5 shows a schematic representation of electrostatic interaction between LA and DOPG at $\mathrm{pH}$ 7.4. LA has a positive charge at $\mathrm{pH} 7.4$ in the solvent environment (isoelectric point of $\mathrm{LA}=9.1$ ), and the hydrophilic group in DOPG shows a negative charge, as shown in Fig. 5. Many reports have observed controlled drug release using electrostatic interactions $(24,25)$. Lim et al. reported that the sustained release of positively charged entecavir was observed after injection by interacting with anionic phospholipid of 1, 2-dipalmitoyl-sn-glycero-3-phosphatidic acid in acidic conditions ( $\mathrm{pH}$ 2.5-4.5) (26). $\mathrm{M}_{\mathrm{DOPG} 12}$ showed a sustained release of LA, suggested that $12 \%$ of DOPG in the 
Fig. 5 Schematic representation of electrostatic interactions between $L A$ and DOPG at $\mathrm{pH} 7.4$ for $M$, $M_{D O P G 12}$ and MDOPG24.

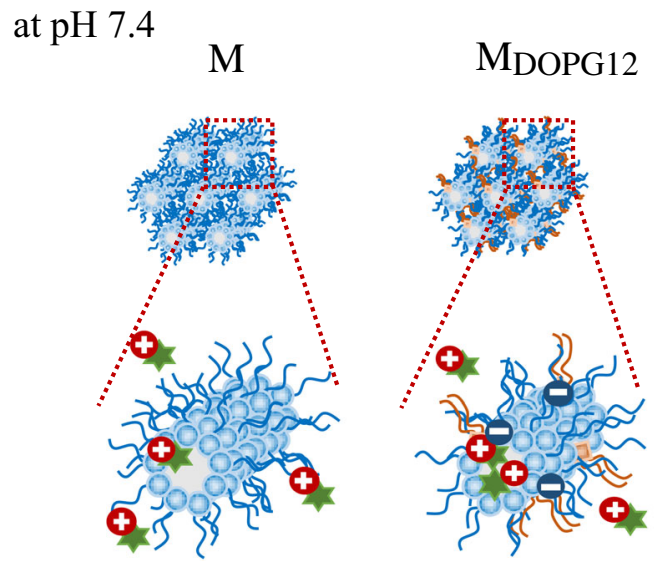

Isoelectric point of $\mathrm{LA}=9.1$

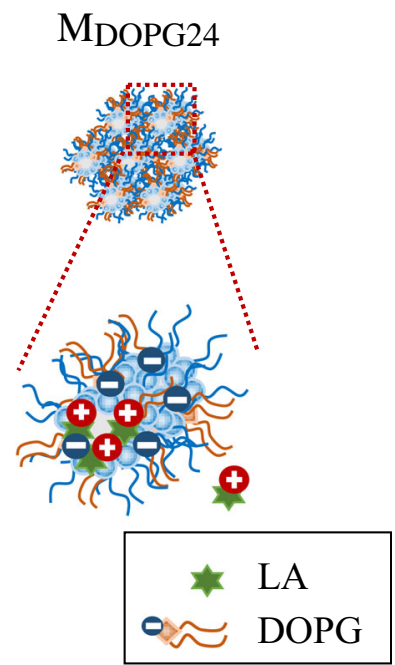

formulation was enough to control the release, and electrostatic interactions between the negatively charged hydrophilic groups of DOPG and positively charged LA may be a reason for the sustained release.

The $\mathrm{M}$ and $\mathrm{M}_{\mathrm{DOPG}}$ formulations exhibited sustained $\mathrm{LA}$ blood levels after s.c. injection. The plasma concentration of LA 21 days after s.c. injection was in the following order; $\mathrm{M}_{\mathrm{DOPG} 12}>\mathrm{M}_{\mathrm{DOPG} 24}>\mathrm{M}$, whereas the amount of LA released over 7 days was in the following order; $\mathrm{M}>$ $\mathrm{M}_{\mathrm{DOPG} 12}>\mathrm{M}_{\mathrm{DOPG} 24}$. Unlike the in vitro condition, the water absorption rate into the administered formulation might be low due to body fluid restrictions in in vivo conditions. Relatively prompt NLLC construction changes from the administered formulation occurred in in vitro conditions, which was due to the plentiful existence of water compared with in vivo condition. On the other hand, the construction change in the NLLC structure may occur gradually from the peripheral part of the administered formulation even in in vivo conditions, and it may take time to construct the NLLC structure in the deeper part of the formulation. This might be a reason for the discrepancy in the observed difference between the release rate observed in the in vitro and in vivo profiles. When LA release rates of $\mathrm{M}, \mathrm{M}_{\mathrm{DOPG} 12}$ and $\mathrm{M}_{\mathrm{DOPG} 24}$ were calculated from in vitro release profiles with four successive points from the last (48 h, $72 \mathrm{~h}, 128 \mathrm{~h}$, and $168 \mathrm{~h}$ ), the release rate was following order; $\mathrm{M}\left(0.46 \mathrm{~h}^{-1}\right)>\mathrm{Mog}_{\text {DOPG24 }}$ $\left(0.44 \mathrm{~h}^{-1}\right)>\operatorname{Mog}_{\text {DOPG } 12}\left(0.40 \mathrm{~h}^{-1}\right)$. Mog12 showed the highest blood concentration of LA, and Met displayed the lowest one among the formulations. Therefore, the LA release rate after sufficient time passed to construct the NLLC structure in in vitro experiment was considered to be reflected in the in vivo results. A robust correlation of both the rate of drug release obtained from in vitro release experiments and the real rate of drug release into the body is still difficult to determine for depot formulations (8). Therefore, the establishment of an in vivo-in vitro correlation (IVIVC) might help in developing depot formulations.

The calculated $F$ value obtained from $\mathrm{M}_{\text {DOPG } 12}$ was about $90 \%$, and the blood concentration of LA was still detectable 21 days after administration. $A U C_{\text {infinity }}$ was calculated by summation of $A U C_{21 \text { days }}+$ Conc.21days $/ k_{\text {el }}$, where Conc.21days represents the plasma concentration at 21 days after administration and $k_{\mathrm{el}}$ is the elimination rate constant. The estimated $F$ with calculated $A U C_{\text {infinity }}$ reached almost $100 \%$. The same calculation was applied to the $\mathrm{M}$ formulation; the estimated $F$ was about $66 \%$. On the other hand, the $F$ value obtained after s.c. injection of LA was about $48 \%$. Instability of LA in the skin tissue was reported, with over 90\% of LA degraded within $120 \mathrm{~min}$ (15). LA was predominantly metabolized by hydrolysis (27). Several kinds of enzymes are present in the skin, such as peptidases (28). Therefore, the lower $F$ value obtained after s.c. injection of LA solution may be ascribed to hydrolysis by such enzymes. On the other hand, an NLLC structure has the potential to protect against enzymatic degradation $(29,30)$. Thus, the stability of LA in skin may be also contributed to by higher $F$ values. The stability of peptides and proteins entrapped in the formulation is one of the most critical considerations for sustained drug delivery. It has been reported that protein instability is attributed to local $\mathrm{pH}$ acidification caused by poly(lactic-co-glycolic acid) degradation (31). Therefore, lipid-based depot formulations using NLLC might be a promising alternative to PLGA formulations. Throughout in vivo experiment over 21 days, no severe toxicities were detected (no weight loss, no respiratory failure and no inflammation at the administrated site). Further safety evaluation experiments should be done to show the usefulness of MGE-based depot formulation. 


\section{CONCLUSION}

The controlled release of LA from a C17MGE depot was possible by adding various phospholipids. In particular, $\mathrm{M}_{\text {DOPG12 }}$ showed a highly sustained LA release and improved absolute bioavailability. Recently, hollow microneedles have been developed to achieve direct intradermal delivery of drugs. However, the relative bioavailability of LA from dissolving microneedles was low due to the instability of LA in the skin (25). Therefore, a combination of a microneedle device and NLLC formulation composed of C17MGE may improve instability and accomplish controlled release of LA. The NLLC formulation used in this study might be used as a depot base with controlled drug release, although further investigations are needed.

Acknowledgments and Disclosures. Part of this work was supported by the NIMS microstructural characterization platform (NMCP) as a program of the "Nanotechnology Platform" from the Ministry of Education, Culture, Sports, Science and Technology (MEXT), Japan, Grant Number JPMXP09S20NR0004. We are grateful to Miss Fujita at NMCP for sample observation with the cryo-TEM. The authors declare that there is no conflict of interest.

\section{REFERENCES}

1. Muramatsu N, Akiyama H. Japan: super-aging society preparing for the future. Gerontologist. 2011;51:425-32.

2. Zabara A, Mezzenga R. Controlling molecular transport and sustained drug release in lipid-based liquid crystalline mesophases. J Control Release. 2014;188:31-43.

3. Kulkarni GV, Wachter W, Iglesias-Salto G, Engelskirchen S, Ahualli S. Monoolein: a magic lipid? Phys Chem Chem Phys. 2011;13:3004-21.

4. Evenbratt H, Ström A. Phase behavior, rheology, and release from liquid crystalline phases containing combinations of glycerol monooleate, glyceryl monooleyl ether, propylene glycol, and water. RSC Adv. 2017;7:32966-73.

5. Boge L, Bysell H, Ringstad L, Wennman D, Umerska A, Cassisa V, et al. Lipid-based liquid crystals as carriers for antimicrobial peptides: phase behavior and antimicrobial effect. Langmuir. 2016;32: 4217-28.

6. Ki M-H, Lim J-L, Ko J-Y, Park S-H, Kim J-E, Cho H-J, et al. A new injectable liquid crystal system for one month delivery of leuprolide. J Control Release. 2014;185:62-70.

7. Okada H. One- and three-month release injectable microspheres of the LH-RH superagonist leuprorelin acetate. Adv Drug Deliv Rev. 1997;28:43-70.

8. Sequeira JAD, Santos AC, Serra J, Veiga F, Ribeiro AJ. Poly(lacticco -glycolic acid) (PLGA) matrix implants. anostructures for the Engineering of Cells, Tissues and Organs. Elsevier; 2018. p. 375402

9. Kim D-H, Jahn A, Cho S-J, Kim JS, Ki M-H, Kim D-D. Lyotropic liquid crystal systems in drug delivery: a review. J Pharm Investig. 2015;45:1-11.
10. van't Hag L, Gras SL, Conn CE, Drummond CJ. Lyotropic liquid crystal engineering moving beyond binary compositional space ordered nanostructured amphiphile self-assembly materials by design. Chem Soc Rev. 2017;46:2705-31.

11. Kadhum WR, Hada T, Hijikuro I, Todo H, Sugibayashi K. Development and optimization of orally and topically applied liquid crystal drug formulations. J Oleo Sci. 2017;66:939-50.

12. Kadhum WR, Sekiguchi S, Hijikuro I, Todo H, Sugibayashi K. A novel chemical enhancer approach for transdermal drug delivery with C17-monoglycerol ester liquid crystal-forming lipid. J Oleo Sci. 2017;66:443-54.

13. See GL, Arce F, Dahlizar S, Okada A, Fadli MFBM, Hijikuro I, et al. Enhanced nose-to-brain delivery of tranilast using liquid crystal formulations. J Control Release. 2020;325:1-9.

14. Adjei A, Love S, Johnson E, Diaz G, Greer J, Haviv F, et al. Effect of formulation adjuvants on gastrointestinal absorption of leuprolide acetate. J Drug Target. 1993;1:251-8.

15. Ito Y, Murano H, Hamasaki N, Fukushima K, Takada K. Incidence of low bioavailability of leuprolide acetate after percutaneous administration to rats by dissolving microneedles. Int J Pharm. 2011;407:126-31.

16. Okada A, Todo H, Hijikuro I, Itakura S, Sugibayashi K. Controlled release of a model hydrophilic high molecular weight compound from injectable non-lamellar liquid crystal formulations containing different types of phospholipids. Int J Pharm. 2020;577: 118944.

17. Sugibayashi K, Yamamoto N, Itakura S, Okada A, Hijikuro I, Todo H. Development of spray formulations applied to the oral mucosa using non-lamellar liquid crystal-forming lipids. Chem Pharm Bull. 2020;68:1025-33.

18. Okawara M, Hashimoto F, Todo H, Sugibayashi K, Tokudome Y. Effect of liquid crystals with cyclodextrin on the bioavailability of a poorly water-soluble compound, diosgenin, after its oral administration to rats. Int J Pharm. 2014;472:257-61.

19. Fong W-K, Hanley T, Boyd BJ. Stimuli responsive liquid crystals provide 'on-demand' drug delivery in vitro and in vivo. J Control Release. 2009; 135:218-26.

20. Báez-Santos YM, Otte A, Mun EA, Soh B-K, Song C-G, Lee Y, et al. Formulation and characterization of a liquid crystalline hexagonal mesophase region of phosphatidylcholine, sorbitan monooleate, and tocopherol acetate for sustained delivery of leuprolide acetate. Int J Pharm. 2016;514:314-21.

21. Yaghmur A, de Campo L, Sagalowicz L, Leser ME, Glatter O. Control of the internal structure of MLO-based isasomes by the addition of diglycerol Monooleate and soybean phosphatidylcholine. Langmuir. 2006;22:9919-27.

22. Huang Y, Gui S. Factors affecting the structure of lyotropic liquid crystals and the correlation between structure and drug diffusion. RSC Adv. 2018;8:6978-87.

23. Lynch ML, Ofori-Boateng A, Hippe A, Kochvar K, Spicer PT. Enhanced loading of water-soluble actives into bicontinuous cubic phase liquid crystals using cationic surfactants. J Colloid Interface Sci. 2003;260:404-13.

24. Negrini R, Sánchez-Ferrer A, Mezzenga R. Influence of electrostatic interactions on the release of charged molecules from lipid cubic phases. Langmuir. 2014;30:4280-8.

25. Lynch ML, Ofori-Boateng A, Hippe A, Kochvar K, Spicer PT. Enhanced loading of water-soluble actives into bicontinuous cubic phase liquid crystals using cationic surfactants. J Colloid Interface Sci. 2003;260:404-13.

26. Lim J-L, Ki M-H, Joo MK, An S-W, Hwang K-M, Park E-S. An injectable liquid crystal system for sustained delivery of entecavir. Int J Pharm. 2015;490:265-72.

27. Tuncer Degim I, Celebi N. Controlled delivery of peptides and proteins. Curr Pharm Des. 2007;13:99-117. 
28. Todo H. Transdermal permeation of drugs in various animal species. Pharmaceutics. 2017;9:33.

29. Clogston J, Craciun G, Hart DJ, Caffrey M. Controlling release from the lipidic cubic phase by selective alkylation. J Control Release. 2005;102:441-61.

30. Mohammady SZ, Pouzot M, Mezzenga R. Oleoylethanolamidebased lyotropic liquid crystals as vehicles for delivery of amino acids in aqueous environment. Biophys J. 2009;96:1537-46.
31. Estey T, Kang J, Schwendeman SP, Carpenter JF. BSA degradation under acidic conditions: a model for protein instability during release from PLGA delivery systems. J Pharm Sci. 2006;95:162639.

Publisher's Note Springer Nature remains neutral with regard to jurisdictional claims in published maps and institutional affliations.? 